\title{
ON THE ABSOLUTE SUMMABILITY OF A FOURIER SERIES AND ITS CONJUGATE SERIES
}

FU CHENG HSIANG

1. Let $f(x)$ be a function integrable in the Lebesgue sense and periodic with period $2 \pi$. Let

$$
f(x) \sim \frac{1}{2} a_{0}+\sum_{n=1}^{\infty}\left(a_{n} \cos n x+b_{n} \sin n x\right) .
$$

Let its conjugate series be

$$
\sum_{n=1}^{\infty}\left(b_{n} \cos n x-a_{n} \sin n x\right) .
$$

We denote by $\sigma_{n}^{\alpha}(x)$ the $n$th Cesàro mean of order $\alpha$ of the Fourier series of $f(x)$. If the series

$$
\sum_{n=1}^{\infty}\left|\sigma_{n}^{\alpha}(x)-\sigma_{n-1}^{\alpha}(x)\right|
$$

converges, then we say that the Fourier series of $f(x)$ is absolutely summable $(C, \alpha)$ or summable $|C, \alpha|$ at the point $x$.

Further, we write

$$
\begin{aligned}
W(\theta, t) & =f(\theta+t)-f(\theta), \\
W_{p}(t) & =\left(\frac{1}{2 \pi} \int_{-\pi}^{\pi}|f(\theta+t)-f(\theta)|^{p} d \theta\right)^{1 / p} .
\end{aligned}
$$

The following two theorems were obtained by Chow [2, p. 440] as corollaries of theorems of Tsuchikura $[3 ; 4]$ and Wang $[5]$ respectively.

TheOREM A. If

$$
W(\theta, t)=O\left\{\left(\log \frac{1}{|t|}\right)^{-(1+\delta)}\right\}
$$

uniformly in $\theta$, for some $\delta>0$, then the Fourier series of $f(x)$ and its conjugate series are summable $|C, \alpha|$ everywhere for $\alpha>1 / 2$.

TheOREM B. If

Received by the editors January 1, 1959 and, in revised form, March 20, 1959. 


$$
W_{2}(t)=O\left\{\left(\log \frac{1}{|t|}\right)^{-(1+\delta)}\right\}
$$

for some $\delta>0$, then the Fourier series of $f(x)$ and its conjugate series are summable $|C, \alpha|$ almost everywhere for $\alpha>1 / 2$. ing

As a generalization of Theorem $B$, Chow has established the follow-

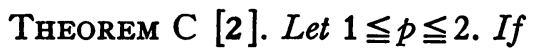

$$
W_{p}(t)=O\left\{\left(\log \frac{1}{|t|}\right)^{-(1+\delta)}\right\}
$$

or, more generally, if

$$
\int_{-\pi}^{\pi} \frac{W_{p}(t)}{|t|} d t<\infty
$$

then the Fourier series of $f(x)$ and its conjugate series are both summable $|C, \alpha|$ almost everywhere for $\alpha>1 / p$.

2. We write

$$
\begin{aligned}
\omega(\theta, t) & =\int_{0}^{t}(f(\theta+u)-f(\theta)) d u, \\
\Omega_{p}(t) & =\left(\frac{1}{2 \pi} \int_{-\pi}^{\pi}\left|\int_{0}^{t}(f(\theta+u)-f(\theta)) d u\right|^{p} d \theta\right)^{1 / p} .
\end{aligned}
$$

We first prove that the condition

$$
\int_{-\pi}^{\pi} W_{p}(t) d t<\infty
$$

involves the boundedness of $\Omega_{p}(t)$. We have, by Minkowski's inequality, if $0 \leqq t \leqq \pi$,

$$
\begin{aligned}
\Omega_{p}(t) & \leqq \int_{0}^{t}\left(\frac{1}{2 \pi} \int_{-\pi}^{\pi}|f(\theta+u)-f(\theta)|^{p} d \theta\right)^{1 / p} d u \\
& =\int_{0}^{t} W_{p}(u) d u \\
& \leqq \int_{-\pi}^{\pi} W_{p}(u) d u \\
& <\infty .
\end{aligned}
$$


The same conclusion can be drawn for $-\pi \leqq t \leqq 0$ if we write

$$
\Omega_{p}(t)=\left(\frac{1}{2 \pi} \int_{-\pi}^{\pi}\left|\int_{t}^{0}(f(\theta+u)-f(\theta)) d u\right|^{p} d \theta\right)^{1 / p}
$$

and operate in a similar way.

Moreover, we can show that the condition (iii) implies, in fact, the following condition

$$
\int_{-\pi}^{\pi} \frac{\Omega_{p}(t)}{t^{2}} d t<\infty
$$

We have, for $0 \leqq t \leqq \pi$,

$$
\begin{aligned}
\int_{0}^{\pi} \frac{\Omega_{p}(t)}{t^{2}} d t & \leqq \int_{0}^{\pi} \frac{d t}{t^{2}} \int_{0}^{t} W_{p}(u) d u \\
& =\int_{0}^{\pi} W_{p}(u) d u \int_{u}^{\pi} \frac{d t}{t^{2}} \text { (by Fubini's theorem) } \\
& \leqq \int_{0}^{\pi} \frac{W_{p}(u)}{u} d u \\
& \leqq \int_{-\pi}^{\pi} \frac{W_{p}(u)}{|u|} d u \\
& <\infty
\end{aligned}
$$

A similar argument gives the same conclusion for negative $t$.

We ask whether the condition (iii) of Chow's theorem can be replaced by the weaker conditions (vi) and (vii). Our answer is positive. In the present note, we improve Theorem $\mathrm{C}$ as follows:

THEOREM. Let $1 \leqq p \leqq 2$. If (vi) and (vii) are satisfied, then the Fourier series of $f(x)$ and its conjugate series are summable $|C, \alpha|$ for $\alpha>1 / p$.

3. The proof of the theorem is based on complex-variable methods. Let

$$
c_{0}=1 / 2, \quad c_{n}=a_{n}-i b_{n} \quad(n \geqq 1) .
$$

Then the function

$$
F(z)=\sum_{n=0}^{\infty} c_{n} z^{n}=\sum_{n=0}^{\infty} c_{n} r^{n} e^{i n \theta}
$$

is regular in the circle $|z|=r<1$. The following lemma is due to Chow: 
1960]

ABSOLUTE SUMMABILITY OF A FOURIER SERIES

35

LemMa 1. The Fourier series of $f(x)$ and its conjugate series are summable $|C, \alpha|$ almost everywhere on the unit circle for $\alpha>1 / p$ provided that

$$
\int_{0}^{r} M_{p}\left(\rho, F^{\prime}\right) d \rho=\int_{0}^{r}\left(\frac{1}{2 \pi} \int_{-\pi}^{\pi}\left|F^{\prime}\left(\rho e^{i \theta}\right)\right| p d \theta\right)^{1 / p} d \rho
$$

is bounded as $r \rightarrow 1-0$.

Now,

$$
\begin{aligned}
F^{\prime}\left(\rho e^{i \theta}\right)= & \frac{1}{\pi} \int_{-\pi}^{\pi} \frac{f(t) e^{i t}}{\left(e^{i t}-\rho e^{i \theta}\right)^{2}} d t \\
= & \frac{e^{-i \theta}}{\pi} \int_{-\pi}^{\pi} \frac{f(\theta+t) e^{i t}}{\left(e^{i t}-\rho\right)^{2}} d t \\
= & \frac{e^{-i \theta}}{\pi} \int_{-\pi}^{\pi} \frac{e^{i t}}{\left(e^{i t}-\rho\right)^{2}}(f(\theta+t)-f(\theta)) d t \quad[2, \mathrm{p} .441] \\
= & \frac{e^{i \theta}}{\pi}\left[\frac{e^{-i t}}{\left(e^{i t}-\rho\right)^{2}} \omega(\theta, t)\right]_{-\pi}^{\pi}-\frac{e^{-i \theta}}{\pi} \int_{-\pi}^{\pi} \omega(\theta, t) \frac{\partial}{\partial t}\left(\frac{e^{i t}}{\left(e^{i t}-\rho\right)^{2}}\right) d t \\
= & -\frac{1}{\pi} \frac{e^{-i \theta}}{(1+\rho)^{2}} \omega(\theta, \pi)+\frac{1}{\pi} \frac{e^{-i \theta}}{(1+\rho)^{2}} \omega(\theta,-\pi) \\
& -\frac{e^{-i \theta}}{\pi} \int_{-\pi}^{\pi} \omega(\theta, t) \frac{\partial}{\partial t}\left(\frac{e^{i t}}{\left(e^{i t}-\rho\right)^{2}}\right) d t \\
= & \phi_{1}(\theta)+\phi_{2}(\theta)+\phi_{3}(\theta),
\end{aligned}
$$

say. We have

$$
\begin{aligned}
&(2 \pi)^{1 / p} M_{p}\left(\rho, F^{\prime}\right)=\left(\int_{-\pi}^{\pi}\left|F^{\prime}\left(\rho e^{i \theta}\right)\right|^{p} d \theta\right)^{1 / p} \\
&=\left(\int_{-\pi}^{\pi}\left|\phi_{1}(\theta)+\phi_{2}(\theta)+\phi_{3}(\theta)\right| p d \theta\right)^{1 / p} \\
& \leqq \\
&\left(\int_{-\pi}^{\pi}\left|\phi_{1}(\theta)\right|^{p} d \theta\right)^{1 / p}+\left(\int_{-\pi}^{\pi}\left|\phi_{2}(\theta)\right|^{p} d \theta\right)^{1 / p} \\
&+\left(\int_{-\pi}^{\pi}\left|\phi_{3}(\theta)\right|^{p} d \theta\right)^{1 / p}
\end{aligned}
$$

by Minkowski's inequality. 


$$
\begin{aligned}
\left(\int_{-\pi}^{\pi}\left|\phi_{1}(\theta)\right| p d \theta\right)^{1 / p} & \leqq \frac{1}{\pi} \frac{1}{(1+\rho)^{2}}\left(\int_{-\pi}^{\pi}|\omega(\theta, \pi)| p d \theta\right)^{1 / p} \\
& =2^{1 / p} \pi^{1 / p-1} \frac{\Omega_{p}(\pi)}{(1+\rho)^{2}} \\
& =O\left(\frac{1}{(1+\rho)^{2}}\right)
\end{aligned}
$$

A similar argument gives

$$
\left(\int_{-\pi}^{\pi}\left|\phi_{2}(\theta)\right|^{p} d \theta\right)^{1 / p}=O\left(\frac{1}{(1+\rho)^{2}}\right)
$$

Finally, we have

$$
\begin{aligned}
& \left(\int_{-\pi}^{\pi}\left|\phi_{3}(\theta)\right| p d \theta\right)^{1 / p} \\
& \quad=\frac{1}{\pi}\left(\int_{-\pi}^{\pi}\left|\int_{-\pi}^{\pi} \omega(\theta, t) \frac{\partial}{\partial t}\left(\frac{e^{i t}}{\left(e^{i t}-\rho\right)^{2}}\right) d t\right|^{p} d \theta\right)^{1 / p} \\
& \quad \leqq \\
& \quad \frac{1}{\pi} \int_{-\pi}^{\pi}\left(\int_{-\pi}^{\pi}\left|\omega(\theta, t) \frac{\partial}{\partial t}\left(\frac{e^{i t}}{\left(e^{i t}-\rho\right)^{2}}\right)\right|^{p} d \theta\right)^{1 / p} d t \\
& \quad=\frac{1}{\pi} \int_{-\pi}^{\pi}\left|\frac{\partial}{\partial t}\left(\frac{e^{i t}}{\left(e^{i t}-\rho^{2}\right)}\right)\right|\left(\int_{-\pi}^{\pi}|\omega(\theta, t)| p d \theta\right)^{1 / p} d t \\
& =2^{1 / p} \pi^{1 / p-1} \int_{-\pi}^{\pi} \Omega_{p}(t) \frac{1}{1-2 \rho \cos t+\rho^{2}}\left(\frac{1+2 \rho \cos t+\rho^{2}}{1-2 \rho \cos t+\rho^{2}}\right)^{1 / 2} d t \\
& =2^{1 / p} \pi^{1 / p-1}\left(\int_{-\pi}^{0}+\int_{0}^{\pi}\right) \\
& =2^{1 / p} \pi^{1 / p-1}\left(I_{1}+I_{2}\right) .
\end{aligned}
$$

We write, for $0<r<1$,

$$
\begin{aligned}
I_{2} & =\int_{0}^{1-r}+\int_{1-r}^{\pi / 2}+\int_{\pi / 2}^{\pi} \\
& =I_{3}(\rho)+I_{4}(\rho)+I_{5}(\rho),
\end{aligned}
$$

say, then 
1960]

ABSOLUTE SUMMABILITY OF A FOURIER SERIES

37

$$
\begin{aligned}
& \int_{0}^{r} I_{3}(\rho) \mathrm{d} \rho \leqq 2 \int_{0}^{r}\left(\int_{0}^{1-r} \Omega_{p}(t) \frac{d t}{(1-\rho)^{3}}\right) d \rho \\
&=2 \int_{0}^{1-r} \Omega_{p}(t)\left(\int_{0}^{r} \frac{d \rho}{(1-\rho)^{3}}\right) d t \\
& \leqq \int_{0}^{1-r} \Omega_{p}(t) \frac{d t}{(1-r)^{2}} \\
& \leqq \int_{0}^{1-r} \frac{\Omega_{p}(t)}{t^{2}} d t . \\
& \int_{0}^{r} I_{4}(\rho) d \rho \\
&=\int_{0}^{r}\left(\int_{1-r}^{\pi / 2} \Omega_{p}(t) \frac{1}{1-2 \rho \cos t+\rho^{2}}\left(\frac{1+2 \rho \cos t+\rho^{2}}{1-2 \rho \cos t+\rho^{2}}\right)\right)^{1 / 2} d t \\
& \leqq 2 \int_{0}^{\pi / 2} \Omega_{p}(t)\left(\int_{0}^{1} \frac{d \rho}{\left(1-2 \rho \cos t+\rho^{2}\right)^{3 / 2}}\right) d t .
\end{aligned}
$$

Now,

$$
\begin{aligned}
\int_{0}^{1} \frac{d \rho}{\left(1-2 \rho \cos t+\rho^{2}\right)^{3 / 2}} & =\int_{0}^{1} \frac{d \rho}{\left(\sin ^{2} t+(\rho-\cos t)^{2}\right)^{3 / 2}} \\
& =\int_{0}^{1} \frac{d(\rho-\cos t)}{\left(\sin ^{2} t+(\rho-\cos t)^{2}\right)^{3 / 2}} \quad(t \text { fixed }) \\
& =\frac{1}{\sin ^{2} t}\left(\sin \frac{t}{2}+\cos t\right) \\
& \leqq 2 / \sin ^{2} t \\
& \leqq \pi^{2} / 2 t^{2}
\end{aligned}
$$

for $0 \leqq t \leqq \pi / 2$. It follows that

$$
\int_{0}^{r} I_{4}(\rho) d \rho \leqq \pi^{2} \int_{0}^{\pi / 2} \frac{\Omega_{p}(t)}{t^{2}} d t .
$$

For $\pi / 2 \leqq t \leqq \pi$, we have

$$
\frac{1}{1-2 \rho \cos t+\rho^{2}}\left(\frac{1+2 \rho \cos t+\rho^{2}}{1-2 \rho \cos t+\rho^{2}}\right)^{1 / 2} \leqq \frac{1}{1-2 \rho \cos t+\rho^{2}} .
$$

Thus, 


$$
\int_{0}^{r} I_{5}(\rho) d \rho \leqq \int_{\pi / 2}^{\pi} \Omega_{p}(t)\left(\int_{0}^{1} \frac{d \rho}{1-2 \rho \cos t+\rho^{2}}\right) d t .
$$

Since, for $\pi / 2 \leqq t \leqq \pi$,

$$
\begin{aligned}
\int_{0}^{1} \frac{d \rho}{1-2 \rho \cos t+\rho^{2}} & =\frac{\pi-t}{2 \sin t} \\
& \leqq \pi / 4 \\
& \leqq \frac{\pi^{3}}{4} \frac{1}{t^{2}} .
\end{aligned}
$$

Hence,

$$
\int_{0}^{r} I_{5}(\rho) d \rho \leqq \frac{\pi^{3}}{4} \int_{\pi / 2}^{\pi} \frac{\Omega_{p}(t)}{t^{2}} d t
$$

From the above analysis, we obtain

$$
\int_{0}^{r} I_{2}(\rho) d \rho \leqq A \int_{0}^{\pi} \frac{\Omega_{p}(t)}{t^{2}} d t
$$

Similarly,

$$
\int_{0}^{r} I_{1}(\rho) d \rho \leqq B \int_{-\pi}^{0} \frac{\Omega_{p}(t)}{t^{2}} d t
$$

Combining these two relations, we get finally

$$
\int_{0}^{r} M_{p}\left(\rho, F^{\prime}\right) d \rho \leqq C \int_{-\pi}^{\pi} \frac{\Omega_{p}(t)}{t^{2}} d t+K \int_{0}^{1} \frac{d \rho}{(1+\rho)^{2}},
$$

where $A, B, C$ and $K$ are positive constants. Our theorem is thus completely established.

\section{REFERENCES}

1. H. C. Chow, A further note on the summability of power series on its circle of convergence, Ann. Acad. Sinica Taipei vol. 1 (1954) pp. 559-567.

2. - Some new criteria for the absolute summability of a Fourier series and its conjugate series, J. London Math. Soc. vol. 30 (1955) pp. 439-448.

3. T. Tsuchikura, Absolute Cesdro summability of orthogonal series, Tôhoku Math. J. vol. 5 (1953-1954) pp. 52-66.

4. - Absolute Cesàro summability of orthogonal series II, ibid. pp. 302-313.

5. F. T. Wang, Note on the absolute summability of Fourier series, J. London Math. Soc. vol. 16 (1941) pp. 174-176.

National Taiwan University, Taipei, China 